Article

\title{
Screening of Helicoverpa armigera Mobilome Revealed Transposable Element Insertions in Insecticide Resistance Genes
}

\author{
Khouloud KLAI ${ }^{1,2}$, Benoît CHÉNAIS ${ }^{2}$, Marwa ZIDI ${ }^{1}{ }^{\circledR}$, Salma DJEBBI ${ }^{1}$, Aurore CARUSO ${ }^{2}$, \\ Françoise DENIS ${ }^{2}$, Johann CONFAIS ${ }^{3,4}{ }^{-}$, Myriam BADAWI ${ }^{2}{ }^{-}$, Nathalie CASSE ${ }^{2, *}$ and \\ Maha MEZGHANI KHEMAKHEM $1, *(1)$
}

1 Laboratory of Biochemistry and Biotechnology (LR01ES05), Faculty of Sciences of Tunis, University of Tunis El Manar, Tunis 1068, Tunisia; khouloud.klai@fst.utm.tn (K.K.); marwa.zidi@fst.utm.tn (M.Z.); salma.djebbi@fst.utm.tn (S.D.)

2 EA2160 Mer Molécules Santé, Le Mans Université, 72085 Le Mans, France; Benoit.Chenais@univ-lemans.fr (B.C.); Aurore.Caruso@univ-lemans.fr (A.C.); fdenis@univ-lemans.fr (F.D.); Myriam.Badawi@univ-lemans.fr (M.B.)

3 URGI, INRAE, Université Paris-Saclay, 78026 Versailles, France; johann.confais@inrae.fr

4 Plant Bioinformatics Facility, BioinfOmics, INRAE, Université Paris-Saclay, 78026 Versailles, France

* Correspondence: Nathalie.Casse@univ-lemans.fr (N.C.); maha.mezghani@fst.utm.tn (M.M.K.)

Received: 29 October 2020; Accepted: 8 December 2020; Published: 11 December 2020

Simple Summary: Transposable elements (TEs) are mobile DNA sequences that can copy themselves within a host genome. TE-mediated changes in regulation can lead to massive and rapid changes in expression, responses that are potentially highly adaptive when an organism is faced with a mortality agent in the environment, such as an insecticide. Helicoverpa armigera shows a hight number of reported cases of insecticide resistance worldwide, having evolved resistance against pyrethroids, organophosphates, carbamates, organochlorines, and recently to macrocyclic lactone spinosad and several Bacillus thuringiensis toxins. In the present study, we conducted a TE annotation using combined approaches, and the results revealed a total of 8521 TEs, representing 236,132 copies, covering $12.86 \%$ of the $H$. armigera genome. In addition, we underlined TE insertions in defensome genes and we successfully identified nine TE insertions belonging to the RTE, R2, CACTA, Mariner and hAT superfamilies.

Abstract: The cotton bollworm Helicoverpa armigera Hübner (Lepidoptera: Noctuidae) is an important pest of many crops that has developed resistance to almost all groups of insecticides used for its management. Insecticide resistance was often related to Transposable Element (TE) insertions near specific genes. In the present study, we deeply retrieve and annotate TEs in the H. armigera genome using the Pipeline to Retrieve and Annotate Transposable Elements, PiRATE. The results have shown that the TE library consists of 8521 sequences representing 236,132 TE copies, including 3133 Full-Length Copies (FLC), covering $12.86 \%$ of the H. armigera genome. These TEs were classified as $46.71 \%$ Class I and 53.29\% Class II elements. Among Class I elements, Short and Long Interspersed Nuclear Elements (SINEs and LINEs) are the main families, representing $21.13 \%$ and $19.49 \%$ of the total TEs, respectively. Long Terminal Repeat (LTR) and Dictyostelium transposable element (DIRS) are less represented, with $5.55 \%$ and $0.53 \%$, respectively. Class II elements are mainly Miniature Inverted Transposable Elements (MITEs) (49.11\%), then Terminal Inverted Repeats (TIRs) (4.09\%). Superfamilies of Class II elements, i.e., Transib, P elements, CACTA, Mutator, PIF-harbinger, Helitron, Maverick, Crypton and Merlin, were less represented, accounting for only $1.96 \%$ of total TEs. In addition, we highlighted TE insertions in insecticide resistance genes and we successfully identified nine TE insertions belonging to RTE, R2, CACTA, Mariner and hAT superfamilies. These insertions are hosted in genes encoding cytochrome P450 (CyP450), glutathione S-transferase (GST), and ATP-binding cassette 
(ABC) transporter belonging to the $\mathrm{G}$ and $\mathrm{C} 1$ family members. These insertions could therefore be involved in insecticide resistance observed in this pest.

Keywords: Helicoverpa armigera; transposable elements; insertions sites; insecticide resistance genes

\section{Introduction}

The cotton bollworm, Helicoverpa armigera (Hübner) (Lepidoptera, Noctuidae), is a serious crop pest having a worldwide distribution [1]. This polyphagous insect causes substantial damages to a wide range of hosts, including cotton, maize, sorghum, and tomato [2]. The biological and ecological traits of $H$. armigera, such as high reproduction rate, polyphagy, high mobility and facultative diapause, make it difficult to control [3]. Management of H. armigera attacks rely heavily on the use of chemicals [4]. However, this practice is harmful to the environment and has caused a rapid buildup of insecticide resistance in H. armigera populations [5].

Insecticide resistance in $H$. armigera is widespread and has evolved against most of commonly used insecticides [6]. To survive, pests have developed various mechanisms to resist against toxic compounds. These mechanisms include point mutations resulting in target-site resistance such as knockdown resistance ( $k d r)$, acetylcholinesterase (Ace-1) and receptor sub-unit termed (RDL) mutations, and also metabolic resistance with involvement of several detoxification enzymes [7].

Metabolic detoxification of toxins is the primary strategy occurring in three phases, each with its own set of enzymes or transporters. Cytochrome P450 monooxygenases (P450s) and carboxylesterases (CarE) carry out phase I, glutathione S-transferases (GSTs) and UDP-glycosyltransferases (UGTs) are phase II enzymes, and ATP-binding cassette transporters (ABC) ensure phase III [8-10]. The understanding of resistance mechanisms remains a challenge that next generation sequencing technologies and the increasing number of sequenced genomes can help to address [11].

Transposable Elements (TEs) are ubiquitous components of eukaryotic genomes that are strongly regulated and inactivated by mutations, which keep transposition events relatively rare $[12,13]$. However, because of their ability to replicate, TEs may accumulate in host genomes and generate abundant sites for chromosomal rearrangements, which may have deleterious or beneficial consequences [14]. In addition, TEs can provide a selective advantage through their insertion sites, which can enhance or repress gene expression or can be domesticated as new host gene [14-16]. Thus, TEs are an important source of variability for the genomes of their hosts and are therefore key to understanding their evolution. Indeed, TEs may be involved in the genetic adaptation of organisms such as insects to stressful environments, among which is the acquisition of insecticide resistance [17].

Several studies have shown that insecticide resistance can be associated with TE insertions in specific genes. For example, dichlorodiphenyltrichloroethane (DTT) resistance in Drosophila melanogaster was correlated with the insertion of a Long Terminal Repeat (LTR)-gypsy retroelement into the $5^{\prime}$ region of the cytochrome P450 gene [18,19]. In Helicoverpa zea, several TE insertions in regulatory regions, exons and introns of cytochrome $\mathrm{P} 450$ genes were related with pyrethroid resistance and xenobiotic metabolism [20].

TEs are classified into two major classes depending on the transposition intermediate. Class I, or retroelements, replicate and transpose via an RNA intermediate; while Class II elements, or DNA transposons, are mobilized via a DNA intermediate [21]. According to the classification of Wicker et al. [22], each class is subdivided into orders and superfamilies. Class I elements are further subdivided into long terminal repeat (LTR) retrotransposons and non-LTR retrotransposons. The non-LTR retrotransposons include the long interspersed nuclear elements (LINEs) and the short interspersed nuclear elements (SINEs) as well as the Penelope-Like Elements (PLEs). Class II elements are subdivided into two subclasses. Subclass 1 includes the terminal inverted repeat (TIR) transposons and Crypton-like elements, which cleave both DNA intermediate strands, while subclass 2 elements including Mavericks 
and Helitrons with a single-strand DNA intermediate have a replicative mode of transposition [22-24]. Class II TIRs transposons also include Miniature Inverted-repeat Transposable Elements (MITEs), which are short ( 100 to $800 \mathrm{bp}$ ) non-autonomous truncated versions of autonomous transposable elements. MITEs possess conserved terminal inverted repeats (TIRs $\geq 10 \mathrm{bp}$ ) and a target site duplication (TSDs = 2 10 bp) $[25,26]$.

Annotation of TEs is a challenging task because of their diversity, their repetitive nature and the complexity of their structures, and numerous tools have been designed to identify TEs [27]. In this study, we used the Pipeline to Retrieve and Annotate Transposable Elements (PiRATE) [28] to annotate the mobilome of $H$. armigera and pinpoint TEs inserted in defensome genes.

\section{Material and Methods}

\subsection{Mobilome Annotation}

The H. armigera genome available in GenBank-NCBI (BioProject PRJNA378437) is 337,087 Mb. This genome is assembled in 24,552 contigs and 998 scaffolds corresponding to $23.5 \mathrm{~kb}$ and $1000 \mathrm{~kb}$ N50 length respectively [29].

H. armigera genome assembly and the corresponding raw Illumina data were both submitted to the PiRATE pipeline to search for TEs following three steps [28]. In the first step, putative TE sequences were detected using four approaches. The first approach is a similarity-based detection of TEs using RepeatMasker [30] and TE-HMMER [31]. The second approach is a structure-based detection, using LTRharvest [32], MGEScan-nonLTR [33] and SINE-Finder [34]. The repetitiveness-based detection is the third approach using TE de novo [35] and Repeat Scout [36]. The last approach is a de novo approach using the dnaPipeTE tool [37].

After TE detection, a second step was performed to eliminate redundant sequences and classify the remaining sequences using the PASTEC tool following the Wicker's 80-80-80 rules corresponding to sequences longer than $80 \mathrm{bp}$, sharing more than $80 \%$ sequence identity and over $80 \%$ of their length [22,38]. Two libraries were generated: a "total TEs library" containing the potentially autonomous TEs and the non-autonomous TEs, and a "repeated elements library" containing the uncategorized repeated sequences and the non-TE sequences. Subsequently, two runs of TEannot [35] were performed for each library to generate the final libraries of total TEs and total repeats. To refine the annotation of TE copies in the whole genome, we used the TEannot pipeline from the REPET package v3.0 with TEs sequences of PiRATE step 1 that align at least with one Full-Length Copy (FLC) on the genome assembly $[35,39,40]$.

Finally, a manual curation was released for all annotated TEs to find corresponding families. This analysis was performed by nucleotide Basic Local Alignment Search Tool (BLAST) against Repbase (48,225 TE sequences) and Dfam (6959 sequences) databases using a threshold value of 80\% (Figure 1).

To identify putative MITE sequences, the H. armigera genome assembly was submitted to the MITE Tracker tool [41] (Figure 1). This tool searches for putative inverted repeat sequences ranging from 50 to 800 bp. Subsequently, putative MITEs were aligned and clustered into families by Vsearch [42] based on target sites duplication (TSD) and Terminal Inverted Repeat (TIR) sequences.

\subsection{Search for TE Insertions in Defensome Genes}

Annotated TEs from the H. armigera genome have been extended by $50 \mathrm{~kb}$ both upstream and downstream of their DNA sequences. The nucleotide BLAST was used to find defensome genes in the extended regions using $80 \%$ similarity and $80 \%$ query coverage threshold (Figure 1). 


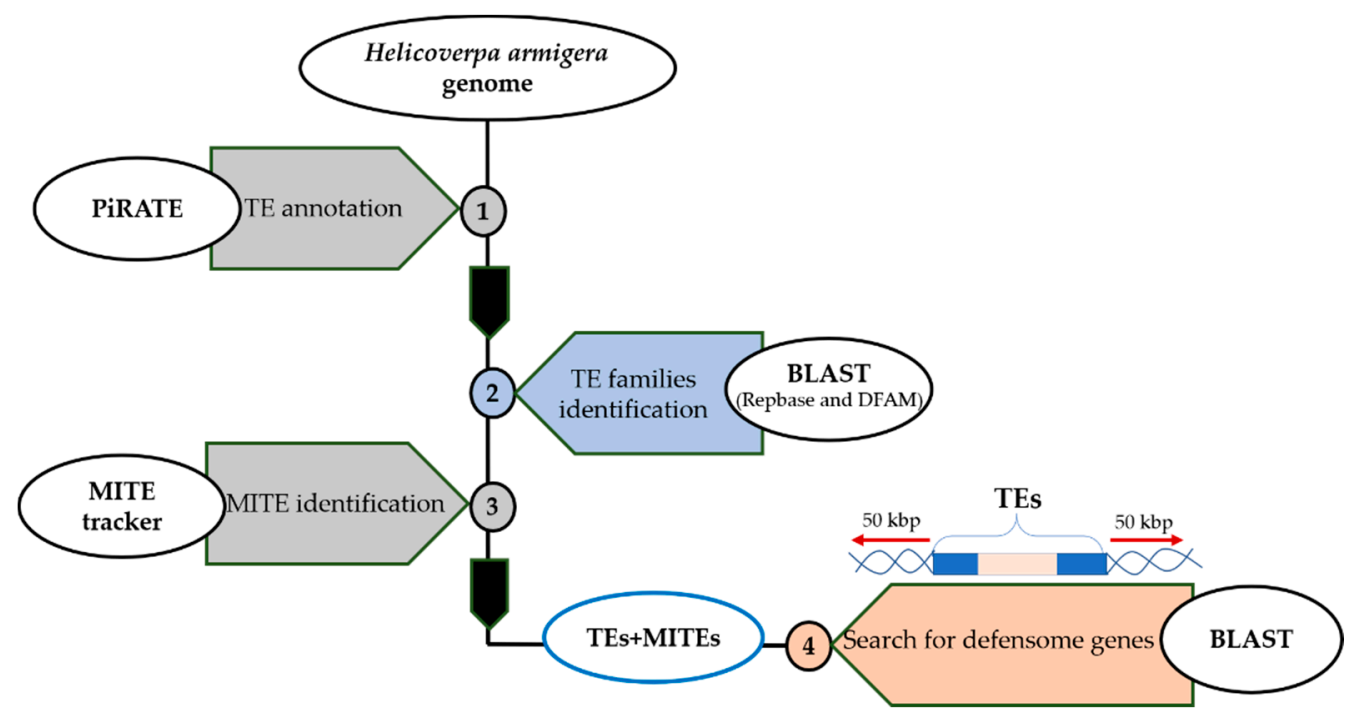

Figure 1. Flowchart for transposable elements annotation and insertion sites identification in the H. armigera genome.

\section{Results}

\subsection{TEs Annotation of the H. armigera Genome}

The screening of TEs in the $H$. armigera genome using different detection tools led to the identification of 100,184 TE candidates after redundance elimination. The classification step generated two libraries: the "total TE library" and the "total repeats library" containing 4336 sequences $(11.36 \%)$ and 5201 sequences $(13.63 \%)$, respectively. Among the TE library, 3133 sequences were identified as FLC belonging to Class I (2720 FLC), Class II (413 FLC) and 61 sequences were undefined (Supplementary File 1). A total of 70,030 sequences was classified as non-TE (41,411 sequences) and unclassified TEs (28,619 sequences). The results revealed that TE sequences cover $12.86 \%(43,349,853 \mathrm{bp})$ of the H. armigera genome and most of the TE sequences belong to Class II elements, accounting for $53.29 \%$ of the total TE content, while Class I elements account for $46.71 \%$.

Among Class I elements, SINEs and LINEs were the main families, representing $21.13 \%$ and $19.49 \%$ of the total TEs, respectively. LTR elements were represented with $5.55 \%$ and Dictyostelium transposable element (DIRS) with only $0.53 \%$. The Class II elements were represented mainly by MITEs and TIRs with $49.11 \%$ and $4.09 \%$, respectively.

To investigate the evolutionary history of TEs in the H. armigera genome, we plotted the distribution of identity values between copies and their representative sequences. Distributions of TE classes showed a peak at $80 \%$ identity for Class I elements, while, for Class II elements, the distribution was linear with a recent burst at $98 \%$ identity (Figure 2).

This analysis revealed that the $H$. armigera genome has undergone a multitude of ancient and recent bursts of different TE superfamilies showing its fluidity. Distributions of TE copies showed three peaks of transposition activity (Figure 3). The first peak is at $65 \%$ divergence involving a burst of the DIRS order. We also noted a second TE burst, particularly for LINE, SINE, LTR, TIR, and Helitron orders at $80 \%$ identity. In addition, the distribution also showed the appearance of MITE elements at $95 \%$ identity, suggesting a recent invasion of the H. armigera genome by these TEs (Figure 3).

\subsubsection{Class I Retrotransposons}

The annotation of Class I retrotransposons in the H. armigera genome allowed for the identification of 3980 sequences representing 186,645 copies belonging to 10 superfamilies (Table 1). 


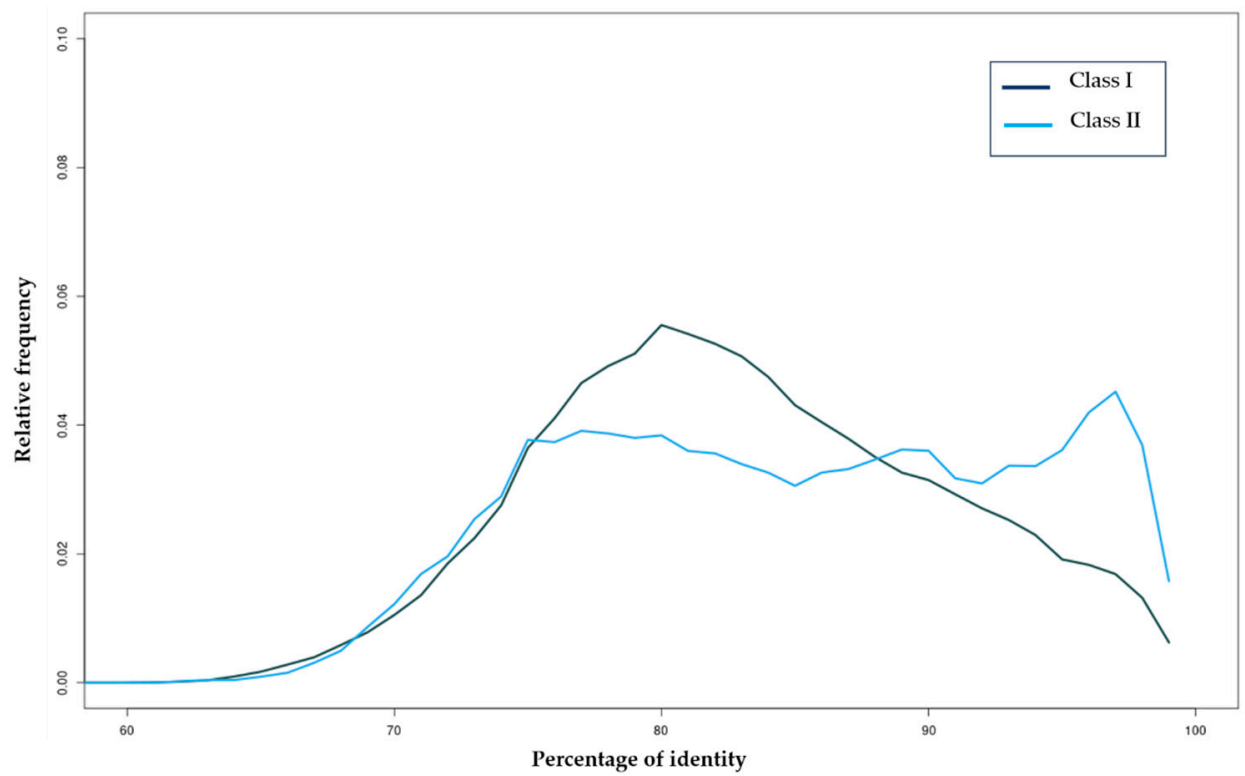

Figure 2. Distribution of sequence identity values between TE copies and TE sequences with at least one full-length copy for Class I and Class II elements. The relative frequencies per percentage of identity of Class I and Class II are represented in different colors.

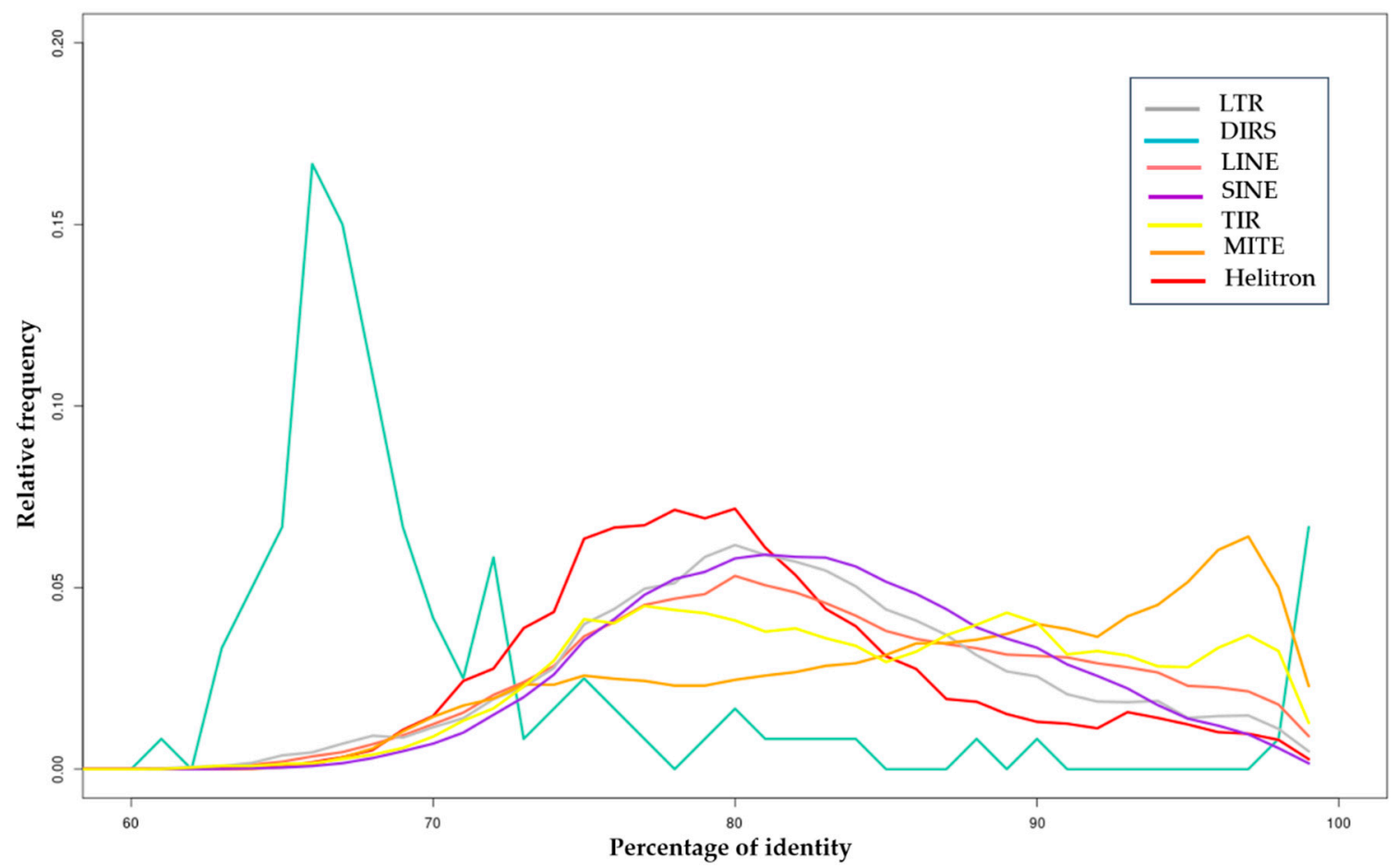

Figure 3. Distribution of sequence identity values between TE copies and TE sequences with at least one FLC. The relative frequencies per percentage of identity of Dictyostelium transposable element (DIRS), Helitron, Long and Short Interspersed Nuclear Elements (LINE and SINE), Long Terminal Repeat (LTR), Miniature Inverted Transposable Element (MITE) and Terminal Inverted Repeat (TIR) orders are represented in different colors. Only the main orders (in terms of copy number) are represented.

From the LTR retrotransposons, 345 TEs were full-length copies (FLC). Gypsy was the most abundant LTR superfamily with 241 sequences and 14,876 copies followed by Bel-Pao (155 sequences) and Copia (77 sequences) with 97 and 67 FLC, respectively. BLAST searches against Repbase and Dfam databases showed that, for all identified LTR sequences, no similarity was found with TEs in databases. 
Table 1. Summary of the identified and annotated TEs in the H. armigera genome.

\begin{tabular}{|c|c|c|c|c|c|c|}
\hline Class & Order & Superfamily & $\begin{array}{c}\text { Total } \\
\text { Sequences }{ }^{1}\end{array}$ & $\begin{array}{c}\text { Full-Length } \\
\text { Copies }^{2}\end{array}$ & $\begin{array}{c}\text { TE } \\
\text { Percentage }\end{array}$ & Copy Number ${ }^{3}$ \\
\hline \multirow{15}{*}{ Class I } & LTR & Gypsy & 241 & 181 & & 14,876 \\
\hline & & Bel pao & 155 & 97 & & 1792 \\
\hline & & Copia & 77 & 67 & & 223 \\
\hline & Total LTR & & 473 & 345 & $5.55 \%$ & 16,891 \\
\hline & DIRS & DIRS & 45 & 38 & & 149 \\
\hline & Total DIRS & & 45 & 38 & $0.53 \%$ & 149 \\
\hline & LINE & Jockey & 716 & 480 & & 44,432 \\
\hline & & RTE & 604 & 543 & & 46,458 \\
\hline & & $\mathbf{I}$ & 172 & 93 & & 16,520 \\
\hline & & $\mathbf{R} 2$ & 169 & 101 & & 10,614 \\
\hline & Total LINE & & 1661 & 1217 & $19.49 \%$ & 118,024 \\
\hline & SINE & tRNA & 1797 & 1120 & & 51,581 \\
\hline & & $5 S$ & 4 & - & & 0 \\
\hline & Total SINE & & 1801 & 1120 & $21.13 \%$ & 51,581 \\
\hline & Total Class I & & 3980 & 2720 & $46.71 \%$ & 186,645 \\
\hline \multirow{10}{*}{$\begin{array}{l}\text { Class II } \\
\text { Subclass I }\end{array}$} & TIR & hAT & 120 & 105 & & 6267 \\
\hline & & Mariner & 106 & 74 & & 2284 \\
\hline & & Piggybac & 41 & 32 & & 6670 \\
\hline & & Transib & 40 & 32 & & 188 \\
\hline & & $\mathbf{P}$ & 15 & 12 & & 6103 \\
\hline & & CACTA & 14 & 11 & & 201 \\
\hline & & Mutator & 6 & 4 & & 477 \\
\hline & & PIF_harbinger & 6 & 5 & & 90 \\
\hline & & Merlin & 1 & 1 & & 30 \\
\hline & Total TIR & & 349 & 276 & $4.09 \%$ & 22,310 \\
\hline \multirow{6}{*}{$\begin{array}{l}\text { Class II } \\
\text { Subclass II }\end{array}$} & Crypton & Crypton & 1 & 1 & & 3 \\
\hline & Helitron & Helitron & 4 & 4 & & 595 \\
\hline & Maverick & Maverick & 2 & 2 & & 14 \\
\hline & MITEs & MITEs & 4185 & 130 & $49.11 \%$ & 26,565 \\
\hline & Total classII & & 4541 & 413 & $53.29 \%$ & 49,487 \\
\hline & Total TEs & & 8521 & 3133 & 100 & 236,132 \\
\hline
\end{tabular}

${ }^{1}$ Representative sequence identified with PiRATE Step 1 with an identity $\leq 80 \%{ }^{2}$ TEs sequences of PiRATE step 1 that align at least with one Full-length Copy (FLC) on the genome assembly ${ }^{3}$ Copies annotated with TEannot REPET package v3.0. Number of undefined transposable elements are not shown in the table.

Regarding LINEs retrotransposons, the most abundant elements belong to the Jockey and RTE superfamilies, with 716 and 604 sequences, respectively, representing 44,432 and 46,458 copies, respectively. The RTE superfamily contains the highest number of FLCs among all annotated TEs (Table 1). According to the BLAST searches, no similarity was found for Jockey elements in databases while 17 RTE sequences showed similarity ranging from $86 \%$ to $100 \%$ with RTE-1_Avan, RTE-2_Hmel_C, RTE-3_DPl, RTE-4_DPl, RTE-5_DPl and Proto2-1_BM families (Table S1).

Concerning SINEs retrotransposons, the tRNA-derived SINE superfamily corresponds to $45.15 \%$ of all Class I TEs with 1797 sequences representing 51,581 copies and 1120 FLCs. Among these sequences, 578 SINEs belong to the HaSE1 family while 85 sequences fell into the HaSE3 family with a similarity ranging from $80 \%$ to $100 \%$ (Table S1). 


\subsubsection{Class II Transposons}

Our results revealed that DNA transposons in the $H$. armigera genome are represented by a total of 4541 sequences representing 49,487 copies belonging to 13 superfamilies (Table 1 ).

Regarding TIR elements, the hAT superfamily was the most abundant, including 120 sequences, among which 105 elements were FLC. BLAST searches against Repbase and Dfam databases revealed high similarity of three hAT elements with the hAT-1_DAN family (Table S1).

The Tc1/mariner superfamily was represented by 106 elements (2284 copies), among which 74 FLC. Research using BLAST has shown that 12 of these elements are distributed among five families-ANM4, nMar-2_Avan, nMar-18_Hmel, Mariner-1_AMel and Mariner-3_BM-exhibiting similarity ranging from $80.25 \%$ to $95.34 \%$ (Table S1).

A total of 41 sequences (6670 copies) belonging to the PiggyBac superfamily was identified in the $H$. armigera genome. Further analysis revealed that only four among the identified sequences showed similarity ranging from $81 \%$ to $99 \%$ with already identified PiggyBac transposons, npiggyBac- 8 and piggyBac-2.

The following superfamilies of Class II elements, i.e., Transib, P elements, CACTA, Mutator, PIF-harbinger, Helitron, Maverick, Crypt-on and Merlin, were less represented in the H. armigera genome corresponding to a total of 7701 copies.

In addition, the MITE tracker allowed for the identification of 4185 putative MITEs corresponding to $43.11 \%$ of all annotated TEs. The analysis of terminal TIRs and TSD sequences allowed for the classification of 3570 MITE sequences (26,565 copies) into seven superfamilies (Table 2). The Tc $1 \backslash$ mariner and PIF-Harbinger superfamilies were the most represented, with 1817 and 1368 MITEs, respectively, followed by the CACTA superfamily with 250 MITEs, then PiggyBac with 93 elements. The hAT, Transib and Maverick superfamilies were represented by only 20, 16 and six MITEs, respectively.

Table 2. Distribution of MITEs identified in the H. armigera genome.

\begin{tabular}{ccccc}
\hline Superfamily & TSD & Number of MITEs & $\begin{array}{c}\text { MITE Length } \\
\mathbf{( b p )}\end{array}$ & $\begin{array}{c}\text { TIR Length } \\
\mathbf{( b p})\end{array}$ \\
\hline Tc1/mariner & TA & 1817 & $50-360$ & $10-21$ \\
\hline PIF-Harbinger & TWA & 1368 & $55-685$ & $15-32$ \\
\hline CACTA & $2-3 \mathrm{bp}$ & 250 & $78-775$ & $10-26$ \\
\hline Piggybac & TTAA & 93 & $50-800$ & $15-31$ \\
\hline hAT & TNNNNA & 20 & $56-260$ & $17-29$ \\
\hline Transib & CNNNG & 16 & $83-386$ & $13-27$ \\
\hline Maverick & 6 bp & 6 & $50-800$ & $10-24$ \\
\hline Other & - & 615 & $50-800$ & $11-35$ \\
\hline
\end{tabular}

\subsection{TE Insertions Scanning in Defensome Genes}

Nucleotide BLAST searches for defensome genes in the regions framing identified TE sequences led to the identification of nine TE insertion sites in seven genes encoding for detoxifying enzymes (Table 3). The involved TEs are members of RTE, R2, CACTA, DIRS, Mariner, and hAT superfamilies (Table 3). Further analysis of TE insertions has shown that five TEs were inserted in four cytochrome P450 genes, an element was retrieved in a GST gene, two were hosted by ABC-G transporter gene and one was inserted in an ABC-C1 transporter gene. 
Table 3. TE insertions in genes encoding for detoxifying enzymes in H. armigera.

\begin{tabular}{|c|c|c|c|c|c|}
\hline Gene Family & Gene Name & $\begin{array}{l}\text { Gene Length } \\
\text { (bp) }\end{array}$ & $\begin{array}{c}\text { Inserted } \\
\text { Element Name }\end{array}$ & $\begin{array}{l}\text { TE Length } \\
\text { (bp) }\end{array}$ & $\begin{array}{l}\text { Insertion } \\
\text { Position }\end{array}$ \\
\hline \multirow[t]{5}{*}{$\begin{array}{c}\text { Cytochrome P450 } \\
\text { (CYP450) }\end{array}$} & $\begin{array}{c}\text { 4g15-like } \\
\text { (LOC110375617) }\end{array}$ & 14,974 & LINE_R2_526 & 4083 & $6483-10,566$ \\
\hline & \multirow{2}{*}{$\begin{array}{c}\text { 4C1-like } \\
\text { (LOC113006340) }\end{array}$} & \multirow{2}{*}{39,707} & LINE_RTE17512 & 620 & $5655-6274$ \\
\hline & & & LINE_RTE84107 & 740 & $7021-7763$ \\
\hline & $\begin{array}{c}\text { 4C1-like } \\
\text { (LOC110381376) } \\
\end{array}$ & 8332 & LTR_CACTA3565 & 1179 & $6899-8066$ \\
\hline & $\begin{array}{c}\text { 6k1-like } \\
\text { (LOC110381042) }\end{array}$ & 15,958 & LINE_jockey2199 & 1629 & $9431-10,438$ \\
\hline $\begin{array}{c}\text { Glutathione } \\
\text { S-transferase (GST) }\end{array}$ & $\begin{array}{c}\text { GST 1-like } \\
\text { (LOC110371343) }\end{array}$ & 4001 & TIR_Mariner2770 & 1304 & 1793-3097 \\
\hline \multirow{3}{*}{$\begin{array}{l}\text { ATP binding cassette } \\
(\mathrm{ABC}) \text { transporter }\end{array}$} & \multirow{2}{*}{$\begin{array}{l}\text { ABC-G member } 20 \\
\text { (LOC110376033) }\end{array}$} & \multirow{2}{*}{96,146} & TIR_Mariner419 & 1085 & $91,998-93,071$ \\
\hline & & & TIR_hAT2824 & 951 & $1486-2436$ \\
\hline & $\begin{array}{l}\text { ABC-C1 (Multidrug } \\
\text { resistance protein } \\
\text { homolog 49-like MRP1) } \\
(\text { LOC110377844) }\end{array}$ & 48,302 & LINE_RTE63004 & 894 & $38,590-39,483$ \\
\hline
\end{tabular}

Six of the inserted TEs have intronic insertion sites and one TE insertion occurred in the first exon of the ABCG transporter member 20 gene (Figure 4 and Figures S1-S7). It should be noted that, for the cytochrome P450 4C1-like (LOC11300634) gene, harboring two LINE insertions, no exon or intron information was retrieved in GenBank.

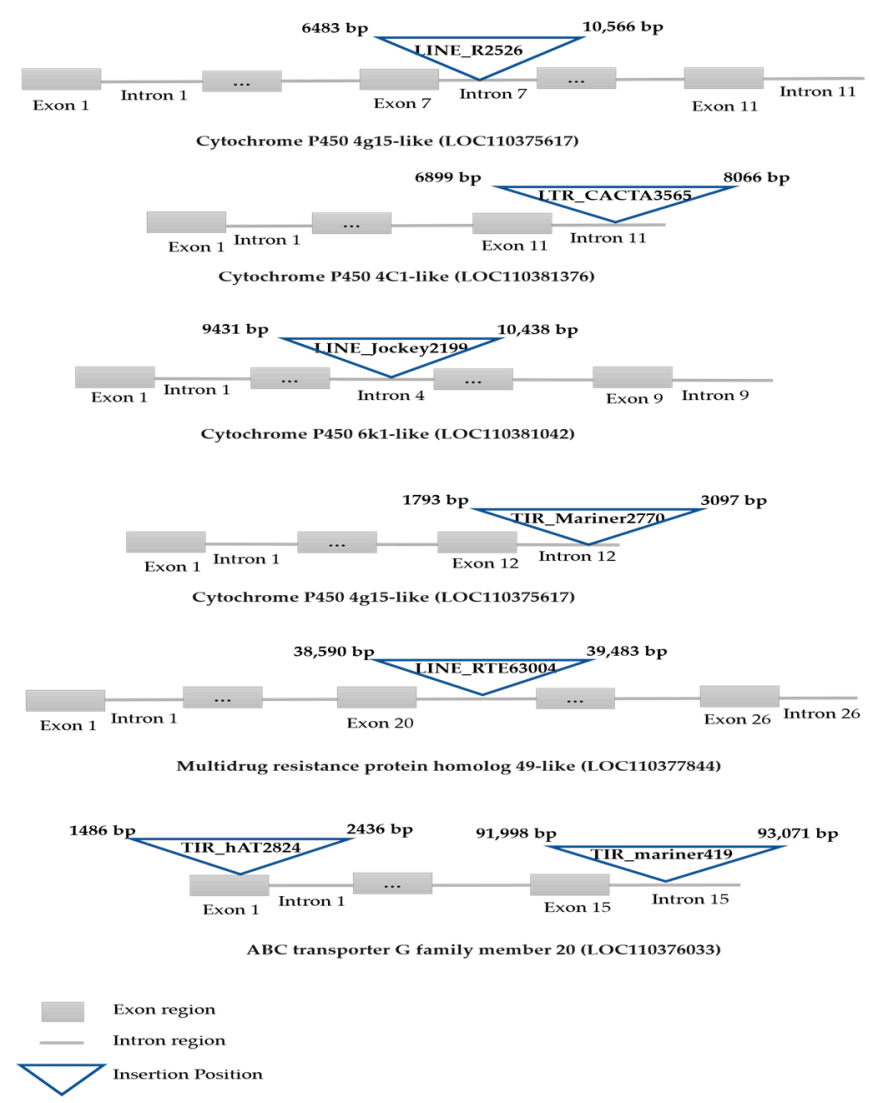

Figure 4. Schematic representation of TE insertion sites in genes encoding for detoxifying enzymes in H. armigera. 


\section{Discussion}

The present study identified TEs present in the genome of H. armigera and searched for their occurrence among the defensome genes of this pest. Characterizing TEs is an important task for non-model organisms and several TE annotation tools have recently been developed for TE characterization in these organisms [43]. In this work, we used the PiRATE pipeline [28], which combines different TE identification tools to detect, classify and annotate TEs into known superfamilies.

The results revealed a total TE content of 4336 sequences, covering $12.86 \%$ of the H. armigera assembly which is much higher than the previous data from Pearce et al. [29], in which Repeat Masker and Repeat Modeller tools allowed for the identification of a TE content representing only $0.88 \%$ of the genome. This confirms the interest of using a pipeline like PiRATE to increase the detection of TEs in a genome.

In other lepidopteran genomes, such as Bombyx mori and Spodoptera frugiperda, about 53\% of TEs were previously identified as Class I and half of these belong to the LINE order [44]. Consistent with these results, $46.71 \%$ of the TEs identified in the $H$. armigera genome were Class I elements and the two most abundant were the SINE and LINE orders. These results suggest that using a combined approach is more specific for short TEs than the use of a single tool.

Some TEs were previously identified in H. armigera by molecular biology methods. In 2008, Sun et al. identified two PiggyBac elements (HaPLE1 and HaPLE2) in the cotton bollworm genome [45]. A few years later, multiple copies of two distinct mariner elements, Hamar1 and Hamar2, were isolated by Wang et al. [46]. All these sequences were retrieved in the current study with a minimum of $88 \%$ identity.

To pinpoint TE insertions in defensome genes, the upstream and downstream regions of TE sequences were scanned and nine insertions have been successfully identified. Among them, five were retrieved in cytochrome P450 genes introns. Cytochrome P450 are among the three main groups of detoxification enzymes used by insects that play crucial roles in the detoxification of most pesticides [47]. In H. armigera, two LINEs (RTE and R2) and a CACTA element were inserted in the P450 4C1-like gene, the expression of which is responsible for the insecticide detoxification [48]. In Aphis gossypii, another LINE, the Jockey element was also identified in cytP450 6K1-like gene intron, putting it as the strongest candidate conferring resistance to thiamethoxam [49]. Two other LINE elements were inserted into the P450 4g15-like gene, but no information for exon or intron regions was retrieved in GenBank for this gene, suggesting that the H. armigera genome needs more annotation. However, the expression of this gene in Aphis gossypii was correlated with imidacloprid resistance [50].

Arthropod ABC transporters also play an important role in metabolite detoxification [10]. Three TEs, which belong to RTE, mariner and $h A T$ superfamilies, were inserted in an ABCG transporter and the gene in the $H$. armigera genome. TE insertions into non-coding regions may be less subject to selection and could be influenced by other mechanisms of control. These insertions could be successfully spliced out during mRNA processing and thus may have no obvious effects on the function of the corresponding defensome gene [51]. Alternatively, they could result in exon skipping, alternative splicing, or alterations in expression profiles if the corresponding IR gene introns contain regulatory sequences, as exemplified by the insertion of the $M u$ element into an intron of the Knotted locus in Maize [51]. Analyses of the transcript and the expression levels of the identified IR genes as well as toxicity bioassay will be necessary to determine the exact effects of the nine identified TE insertions on the expression and function of the identified IR genes, as well as on the fitness of this species in the presence of a stressful environment such as insecticides.

\section{Conclusions}

Genome-wide TE annotation has rarely been performed in H. armigera. This study opens the way to new searches about the role of TEs in the genome evolution of H. armigera and their contribution to the pest adaptation such as insecticide resistance. In the present study, we conducted an accurate TE annotation, and the results reveal a total of 8521 TEs covering $12.86 \%$ (43349853 bp) of the H. armigera 
genome. The annotation of TEs was crossed with insertion sites search in defensome genes. Nine TEs belonging to the RTE, R2, LTR and TIR superfamilies were found to be inserted in CYP450, GST and $\mathrm{ABC}$ transporter genes and their insertion sites were mostly in intronic regions except for a hAT element inserted in the exon region. These results present for the first-time evidence that TEs are present in IR genes of $H$. armigera. However, further studies are necessary to elucidate the functional relationship of TEs with IR genes in H. armigera.

Supplementary Materials: The following are available online at http://www.mdpi.com/2075-4450/11/12/879/s1. File 1. Full-length TEs annotated in the Helicoverpa armigera genome, Table S1: BLAST results of annotated TEs in H. armigera against reference TEs in databases (Repbase and Dfam), Figure S1. Alignment of LINE_R2526 element with Cytochrome P450 4g15-like gene, Figure S2. Alignment of LINE_RTE84107 and LINE_RTE17512 elements with Cytochrome P450 4C-like gene, Figure S3. Alignment of TIR_CACTA3565 element with Cytochrome P450 4C-like gene, Figure S4. Alignment of LINE_Jockey2199 element with Cytochrome P450 6k1-like gene, Figure S5. Alignment of TIR_mariner2770 element with glutathione S-transferase 1-like gene, Figure S6. Alignment of LINE_RTE63004 element with ABC-C1 homolog 49-like gene, Figure S7. Alignment of TIR_mariner419 and TIR_hAT824 elements with ABCG member 20 gene.

Author Contributions: Conceptualization, K.K., M.M.K., A.C., B.C. and N.C.; methodology, K.K., M.Z., M.B., J.C. and F.D.; investigation, data curation, K.K.; writing-original draft preparation, K.K., M.M.K., B.C. and N.C.; writing-review and editing, M.M.K., B.C., N.C., A.C., S.D., F.D.; supervision, M.M.K., B.C. and N.C.; project administration, M.M.K. and N.C.; funding acquisition, M.M.K. and N.C. All authors have read and agreed to the published version of the manuscript.

Funding: This research was funded by PHC-Utique 19G0901 "Hubert Curien Franco-Tunisian partnership" (41703ZJ) and the Tunisian Ministry of Higher Education and Scientific Research and the University of Tunis El Manar.

Conflicts of Interest: The authors declare no conflict of interest.

\section{References}

1. Burgio, G.; Ravaglia, F.; Maini, S.; Bazzocchi, G.G.; Masetti, A.; Lanzoni, A. Mating Disruption of Helicoverpa armigera (Lepidoptera: Noctuidae) on Processing Tomato: First Applications in Northern Italy. Insects 2020, 11, 206. [CrossRef] [PubMed]

2. Tembrock, L.R.; Timm, A.E.; Zink, F.A.; Gilligan, T.M. Phylogeography of the Recent Expansion of Helicoverpa armigera (Lepidoptera: Noctuidae) in South America and the Caribbean Basin. Ann. Entomol. Soc. Am. 2019, 112, 388-401. [CrossRef]

3. Sarate, P.J.; Tamhane, V.A.; Kotkar, H.M.; Ratnakaran, N.; Susan, N.; Gupta, V.S.; Giri, A.P. Developmental and digestive flexibilities in the midgut of a polyphagous pest, the cotton bollworm, Helicoverpa armigera. J. Insect Sci. 2012, 12, 42. [CrossRef] [PubMed]

4. Pereira, F.P.; Reigada, C.; Diniz, A.J.F.; Parra, J.R.P. Potential of Two Trichogrammatidae species for Helicoverpa armigera control. Neotrop. Entomol. 2019, 48, 966-973. [CrossRef]

5. Ansari, M.S.; Moraiet, M.A.; Ahmad, S. Insecticides: Impact on the Environment and Human Health. In Environmental Deterioration and Human Health: Natural and Anthropogenic Determinants; Malik, A., Grohmann, E., Akhtar, R., Eds.; Springer: Dordrecht, The Netherlands, 2014; pp. 99-123. [CrossRef]

6. Srinivas, R.; Udikeri, S.S.; Jayalakshmi, S.K.; Sreeramulu, K. Identification of factors responsible for insecticide resistance in Helicoverpa armigera. Comp. Biochem. Physiol. 2004, 137, 261-269. [CrossRef]

7. Sarwar, M.; Salman, M. Insecticides Resistance in Insect Pests or Vectors and Development of Novel Strategies to Combat Its Evolution. IJBBE 2015, 1, 344-351.

8. Sampath, P.; Murukarthick, J.; Izzah, N.K.; Lee, J.; Choi, H.I.; Shirasawa, K.; Choi, B.S.; Liu, S.; Nou, I.S.; Yang, T.J. Genome-Wide Comparative Analysis of 20 Miniature Inverted-Repeat Transposable Element Families in Brassica rapa and B. oleracea. PLoS ONE 2014, 9, e94499. [CrossRef]

9. De Marco, L.; Sassera, D.; Epis, S.; Mastrantonio, V.; Ferrari, M.; Ricci, I.; Comandatore, F.; Bandi, C.; Porretta, D.; Urbanelli, S. The choreography of the chemical defensome response to insecticide stress: Insights into the Anopheles stephensi transcriptome using RNA-Seq. Sci. Rep. 2017, 7. [CrossRef]

10. Dermauw, W.; van Leeuwen, T. The ABC gene family in arthropods: Comparative genomics and role in insecticide transport and resistance. Insect Biochem. Mol. Biol. 2014, 45, 89-110. [CrossRef] 
11. Su, H.; Gao, Y.; Liu, Y.; Li, X.; Liang, Y.; Dai, X.; Xu, Y.; Zhou, Y.; Wang, H. Comparative transcriptome profiling reveals candidate genes related to insecticide resistance of Glyphodes pyloalis. Bull. Entomol. Res. 2020, 110, 57-67. [CrossRef]

12. Mérel, V.; Boulesteix, M.; Fablet, M.; Vieira, C. Transposable elements in Drosophila. Mob. DNA 2020, 11, 23. [CrossRef] [PubMed]

13. Lavoie, C.A. Transposable Element Content in Non-Model Insect Genomes. Master's Thesis, Mississippi State University, Starkwell, MS, USA, 2020.

14. Chénais, B.; Caruso, A.; Hiard, S.; Casse, N. The impact of transposable elements on eukaryotic genomes: From genome size increase to genetic adaptation to stressful environments. Gene 2012, 509, 7-15. [CrossRef] [PubMed]

15. Bourgeois, Y.; Boissinot, S. On the Population Dynamics of Junk: A Review on the Population Genomics of Transposable Elements. Genes 2019, 10, 419. [CrossRef] [PubMed]

16. Steinberg, C.E.W. Arms Race between Plants and Animals: Biotransformation System in Stress Ecology: Environmental Stress as Ecological Driving Force and Key Player in Evolution; Springer: New York, NY, USA, 2012; pp. 61-105.

17. Le Goff, G.; Hilliou, F. Resistance evolution in Drosophila: The case of CYP6G1. Pest Manag. Sci. 2017, 73, 493-499. [CrossRef]

18. Catania, F.; Kauer, M.O.; Daborn, P.J.; Yen, J.L.; French-Constant, R.H.; Schlötterer, C. World-wide survey of an Accord insertion and its association with DDT resistance in Drosophila melanogaster. Mol. Ecol. 2004, 13, 2491-2504. [CrossRef]

19. Schlenke, T.A.; Begun, D.J. Strong selective sweep associated with a transposon insertion in Drosophila simulans. Proc. Natl. Acad. Sci. USA 2004, 101, 1626-1631. [CrossRef]

20. Chen, S.; Li, X. Transposable elements are enriched within or in close proximity to xenobiotic-metabolizing cytochrome P450 genes. BCM Evol. Biol. 2007, 7, 46. [CrossRef]

21. Finnegan, D.J. Eukaryotic transposable elements and genome evolution. Trends Genet. 1989, 5, $103-107$. [CrossRef]

22. Wicker, T.; Sabot, F.; Hua-Van, A.; Bennetzen, J.L.; Capy, P.; Chalhoub, B.; Flavell, A.; Leroy, P.; Morgante, M.; Panaud, O.; et al. An unified classification system for eukaryotic transposable elements. Nat. Rev. Genet. 2007, 8, 973-982. [CrossRef]

23. Kapitonov, V.V.; Jurka, J. Rolling-Circle Transposons in Eukaryotes. Proc. Natl. Acad. Sci. USA 2001, 98, 8714-8719. [CrossRef]

24. Pritham, E.J.; Putliwala, T.; Feschotte, C. Mavericks, a novel class of giant transposable elements widespread in eukaryotes and related to DNA viruses. Gene 2007, 390, 3-17. [CrossRef] [PubMed]

25. Kuang, H.; Padmanabhan, C.; Li, F.; Kamei, A.; Bhaskar, P.B.; Ouyang, S.; Jiang, J.; Robin Buell, C.; Baker, B. Identification of miniature inverted-repeat transposable elements (MITEs) and biogenesis of their SiRNAs in the Solanaceae: New functional implications for MITEs. Genome Res. 2009, 19, 42-56. [CrossRef] [PubMed]

26. Schrader, L.; Schmitz, J. The impact of transposable elements in adaptive evolution. Mol. Ecol. 2019, 28, 1537-1549. [CrossRef] [PubMed]

27. Yan, H.; Torchiana, F.; Bombarely, A. Guideline for genome transposon annotation derived from evaluation of popular TE identification tools. Preprints 2020, 2020080275. [CrossRef]

28. Berthelier, J.; Casse, N.; Daccord, N.; Jamilloux, V.; Saint-Jean, B.; Carrier, G. A transposable element annotation pipeline and expression analysis reveal potentially active elements in the microalga Tisochrysis lutea. BMC Genom. 2018, 19, 1-14. [CrossRef]

29. Pearce, S.L.; Clarke, D.F.; East, P.D.; Elfekih, S.; Gordon, K.H.J.; Jermiin, L.S.; McGaughran, A.; Oakeshott, J.G.; Papanikolaou, A.; Perera, O.P.; et al. Genomic innovations, transcriptional plasticity and gene loss underlying the evolution and divergence of two highly polyphagous and invasive Helicoverpa pest species. BMC Biol. 2017, 15, 1-30. [CrossRef]

30. Smit, A.F.A.; Hubley, R.; Green, P. Repeat Masker. Unpublished Data. Current Version: Open-4.09 (Dfam:3.0 only) 1996. Available online: http://www.repeatmasker.org (accessed on 15 April 2019).

31. Eddy, S.R. Multiple alignment using hidden Markov models. ISMB 1996, 3, 114-120.

32. Ellinghaus, D.; Kurtz, S.; Willhoeft, U. LTR harvest, an efficient and flexible software for de novo detection of LTR retrotransposons. BMC Bioinform. 2008, 9, 18. [CrossRef]

33. Rho, M.; Tang, H. MGEScan-Non-LTR: Computational identification and classification of autonomous non-LTR retrotransposons in eukaryotic genomes. Nucleic Acids Res. 2009, 37, e143. [CrossRef] 
34. Wenke, T.; Döbel, T.; Sörensen, T.R.; Junghans, H.; Weisshaar, B.; Schmidta, T. Targeted Identification of Short Interspersed Nuclear Element Families Shows Their Widespread Existence and Extreme Heterogeneity in Plant Genomes. Plant Cell 2011, 23, 3117-3128. [CrossRef]

35. Flutre, T.; Duprat, E.; Feuillet, C.; Quesneville, H. Considering Transposable Element Diversification in de Novo Annotation Approaches. PLoS ONE 2011, 6, e16526. [CrossRef] [PubMed]

36. Price, A.L.; Jones, N.C.; Pevzner, P.A. De novo identification of repeat families in large genomes. Bioinformatics 2005, 21 (Suppl. 1), i351-i358. [CrossRef] [PubMed]

37. Goubert, C.; Modolo, L.; Vieira, C.; Moro, C.V.; Mavingui, P.; Boulesteix, M. De Novo Assembly and Annotation of the Asian Tiger Mosquito (Aedes albopictus) Repeatome with DnaPipeTE from Raw Genomic Reads and Comparative Analysis with the Yellow Fever Mosquito (Aedes aegypti). GBE 2015, 7, 1192-1205. [CrossRef] [PubMed]

38. Hoede, C.; Arnoux, S.; Moisset, M.; Chaumier, T.; Inizan, O.; Jamilloux, V.; Quesneville, H. PASTEC: An Automatic Transposable Element Classification Tool. PLoS ONE 2014, 9, e91929. [CrossRef]

39. Quesneville, H.; Bergman, C.M.; Andrieu, O.; Autard, D.; Nouaud, D.; Ashburner, M.; Anxolabehere, D. Combined Evidence Annotation of Transposable Elements in Genome Sequences. PLoS Comput. Biol. 2005, 1, 0166-0175. [CrossRef]

40. Daccord, N.; Celton, J.M.; Linsmith, G.; Becker, C.; Choisne, N.; Schijlen, E.; van de Geest, H.; Bianco, L.; Micheletti, D.; Velasco, R.; et al. High-quality de novo assembly of the apple genome and methylome dynamics of early fruit development. Nat. Genet. 2017, 49, 1099-1106. [CrossRef]

41. Crescente, J.M.; Zavallo, D.; Helguera, M.; Vanzetti, L.S. MITE Tracker: An accurate approach to identify miniature inverted-repeat transposable elements in large genomes. BMC Bioinform. 2018, 19, 348. [CrossRef]

42. Rognes, T.; Flouri, T.; Nichols, B.; Quince, C.; Mahé, F. VSEARCH: A versatile open source tool for metagenomics. PeerJ 2016, 4, 2584. [CrossRef]

43. Goerner-Potvin, P.; Bourque, G. Computational tools to unmask transposable elements. Nat. Rev. Genet. 2018, 19, 688-704. [CrossRef]

44. D'Alençon, E.; Sezutsu, H.; Legeai, F.; Permal, E.; Bernard-Samain, S.; Gimenez, S.; Gagneur, C.; Cousserans, F.; Shimomura, M.; Brun-Barale, A.; et al. Extensive synteny conservation of holocentric chromosomes in lepidoptera despite high rates of local genome rearrangements. Proc. Natl. Acad. Sci. USA 2010, 107, 7680-7685. [CrossRef]

45. Sun, Z.C.; Wu, M.; Miller, T.A.; Han, Z.J. PiggyBac-like elements in cotton bollworm, Helicoverpa armigera (Hübner). Insect Mol. Biol. 2008, 17, 9-18. [CrossRef] [PubMed]

46. Wang, J.; Miller, T.A.; Park, Y. Identification of mariner-like elements belonging to the Cecropia subfamily in two closely related Helicoverpa species. Insect Sci. 2011, 18, 619-628. [CrossRef]

47. Zhu, W.; Yu, R.; Wu, H.; Zhang, X.; Liu, Y.; Zhu, K.Y.; Zhang, J.; Ma, E. Identification and characterization of two CYP9A genes associated with pyrethroid detoxification in Locusta migratoria. Pestic. Biochem. Physiol. 2016, 132, 65-71. [CrossRef] [PubMed]

48. Gong, J.; Cheng, T.; Wu, Y.; Yang, X.; Feng, Q.; Mita, K. Genome-wide patterns of copy number variations in Spodoptera litura. Genomics 2019, 111, 1231-1238. [CrossRef]

49. Langfield, K.L. Characterization of Neonicotinoid Resistance in the Cotton Aphid, Aphis gossypii from Australian Cotton. Ph.D. Thesis, University of Technology Sydney, Sydney, Australia, April 2017.

50. Kim, J.I.; Kwon, M.; Kim, G.H.; Kim, S.Y.; Lee, S.H. Two mutations in nAChR beta subunit is associated with imidacloprid resistance in the Aphis gossypii. J. Asia Pac. Entomol. 2015, 18, 291-296. [CrossRef]

51. Greene, B.; Wako', R.; Hake, S. Mutator Insertions in an Intron of the Maize Knotted1 Gene Result in Dominant Suppressible Mutations. Genetics 1994, 138, 1275-1285.

Publisher's Note: MDPI stays neutral with regard to jurisdictional claims in published maps and institutional affiliations. 